\title{
Molecular movies filmed at intersections
}

\section{Electron diffraction maps atomic motions that result from photoexcitation of $\mathrm{CF}_{3} \mathrm{l}$ mole- cules \\ By Helen H. Fielding}

How molecules respond to ultraviolet (UV) light is not only a fundamental issue, but has relevance in nature (e.g. photosynthesis) and technology (e.g. solar cells). Many spectroscopic methods have been developed for studying photoexcited molecules in the gasphase, free from interactions with any environment, because these conditions allow the intrinsic properties of the molecule to be studied in exquisite detail and to be compared directly with theory. Many spectroscopic methods rely on measuring observables from which it is possible to infer the positions of the individual atoms of the molecule but do not directly measure molecular structure. On $\mathrm{p} . \mathrm{xxx}$ of this issue, Yang et al. (1) report the use of ultrafast gas-phase electron diffraction (GED) and high-level calculations to probe the movements of individual atoms in the prototypical molecule $\mathrm{CF}_{3} \mathrm{l}$ after the absorption of UV light.

When a molecule absorbs UV light, it is promoted to an electronically excited state in which the individual atoms are no longer in their equilibrium positions. Their resulting excess potential energy can be redistributed in a variety of ways. For example, a molecular bond may break, or the atoms may rearrange to form a new molecular structure in which the excited electronic state and another electronic state become degenerate (a conical intersection of potential energy surfaces). Such conical intersections provide particularly efficient "funnels" for transferring molecules from an electronically excited state to a lower lying excited electronic state or back to the ground state.

A molecular bond typically breaks or relax through a conical intersection is a few tens of femtoseconds. Spectroscopic measurements on these time scales became possible in the late 1980s with the advent of femtosecond lasers. Zewail and co-workers carried out pioneering experiments using femtosecond lasers to record spectroscopic signatures of molecular bonds breaking and rearranging. (2). In these experiments, a femtosecond pulse of UV light (pump pulse) initiated a photochemical reaction, and a subsequent femtosecond pulse of light (probe pulse) at a series of precisely

Department of Chemistry, University College London, 20 Gordon Street, London WC1H OAJ, UK. Email: h.h.fielding@ucl.ac.uk timed intervals after the pump probed the structure of the electronically excited molecule.

The information that can be retrieved from pump-probe experiment depends on the spectroscopic measurement being made. Femtosecond time-resolved photoelectron spectroscopy has proved to be a powerful detection technique for probing ultrafast electronic relaxation processes in neutral molecules (3-5) and molecular anions $(6,7)$. The related techniques of femtosecond pumpprobe velocity-map ion imaging (8) and timeresolved photoelectron-photoion imaging (9) have also proved valuable for monitoring the fragments of dissociation reactions. However, these techniques probe the evolution of the electronic wave function or the products of dissociation reactions and rely on complementary quantum chemistry calculations to deduce the corresponding molecular geometries after the absorption of the photon or after dissociation.

Time-resolved $x$-ray and electron diffraction techniques, on the other hand, are now emerging as exciting new techniques for realtime measurement of molecular structure following absorption of UV light (molecular movies) $(10,11)$. Yang et al. used the ultrafast GED setup at the SLAC National Accelerator Laboratory to record a molecular movie of photoexcited $\mathrm{CF}_{3} \mathrm{I}$ molecules. A femtosecond UV pump pulse promoted the molecules to electronically excited states. Ultrashort pulses of electrons were then fired at the photoexcited molecules at a series of precisely timed intervals after the UV pump pulse. The resulting interference patterns arising from electron waves being scattered from the nuclei and electron clouds of the molecule were recorded at each time interval. Each diffraction pattern effectively provides a snapshot of the movie of molecular structures at the time each was recorded.

Yang et al. used a single UV photon to initiate $\mathrm{C}-\mathrm{I}$ bond dissociation on an excited electronic state. This process has been the subject of several femtosecond pump-probe experiments and the electronic states involved and time scale for dissociation are wellcharacterized (12). However, the ultrafast GED measurements could also follow the molecular motions of the $\mathrm{CF}_{3}$ fragment after dissociation (see the figure, bottom). This fragment underwent a repeated opening and closing of the F-C-F bond angle (an umbrella motion). This motion was accompanied by an elongation of the C-F bonds, and there was a delay between the initial F-C-F bond opening and the $\mathrm{C}-\mathrm{F}$ bond elongation.

If the $\mathrm{CF}_{3}$ l molecules absorbed two UV photons, they were excited to a different set of higher lying excited electronic states. Ultrafast GED measurements revealed that the nuclei rearranged in the first $100 \mathrm{fs}$ to the molecular structure associated with a conical intersection between this high lying electronic state and one lying just below it. The population of molecules was observed to bifurcate at the conical intersection (see the figure, top). Some of the population was transferred through the conical intersection to lower lying electronically excited states, and the nuclei returned to their initial geometry (before photoexcitation) after $\sim 500 \mathrm{fs}$. The remaining population underwent a C-I stretching motion with a period of $200 \mathrm{fs}$ on the higher lying excited electronic state, but each time the nuclei returned to the geometry corresponding to the conical intersection, more of the population was transferred down to the lower lying states. This process resulting in quantum mechanical interference patterns on the lower lying electronically excited stare. This is the first time that the molecular structure of a molecule has been measured as it passed through a conical intersection.

The results of Yang et al. show that ultrafast GED can measure molecular structure after photoexcitation of molecules in exquisite detail. Combining the information obtained about the nuclear coordinates with that obtained about the electronic wave function from femtosecond pump-probe spectroscopies and time-averaged experiments has the potential to provide the most stringent tests for theory. This understanding will advance our understanding of photochemistry and photobiology to the point where we can design molecular systems with a particular function from first principles.

REFERENCES:

1. J. Yang, Science $\mathbf{X X X}$, xxxx (2018).

2. A. H. Zewail, Angew. Chem. Int. Ed. 39, 2586 (2000). 
3. V. Blanchet, M. Z. Zgierski, T. Seideman, A. Stolow, Nature 401, 52 (1999).

4. R. Spesyvtsev, O. M. Kirkby, M. Vacher, H. H Fielding, Phys. Chem. Chem. Phys. 14, 9942 (2012).

5. T. Horio et al., J. Chem. Phys. 145, 44306 (2016).

6. C. R. S. Mooney et al., Chem. Sci. 4, 921 (2013).

7. M. A. Yandell, S. B. King, D. M. Neumark, J. Am. Chem. Soc. 135, 2128 (2013).

8. G. M. Roberts et al., J. Am. Chem. Soc. 134, 12578 (2012)

9. O. Geßner et al., Science 311, 219 (2006).

10. M. P. Minitti et al., Phys. Rev. Lett. 114, 255501 (2015).

11. A. A. Ischenko, P. M. Weber, R. J. Dwayne Miller, Chem. Rev. 117, 11066 (2017)

12. W. G. Roeterdink, M. H. M. Janssen, Phys. Chem. Chem. Phys. 4, 601 (2002).

10.1126/science.aat6002

Responding to excitation. Potential energy surfaces of CF3I illustrating the dissociative state accessed using a single UV photon and the higher lying excited states accessed using two UV photons and the conical intersection connecting the higher lying excited electronic states. 